\title{
Recent Developments in the Zimmer Program
}

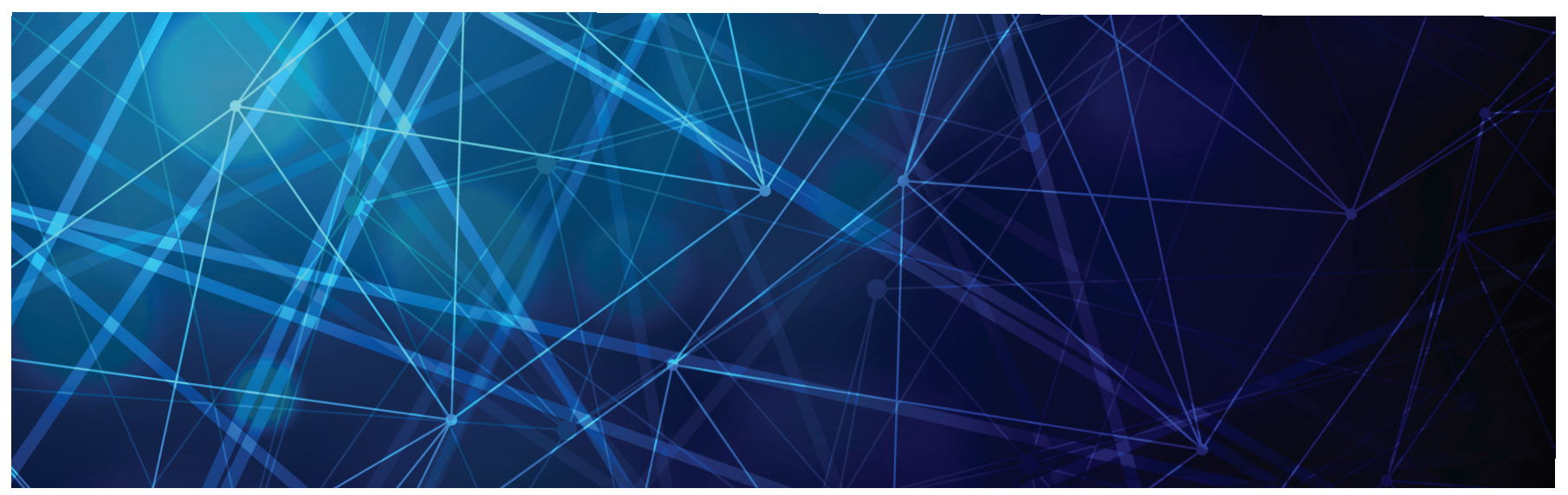

David Fisher

\section{Introduction}

The group of diffeomorphisms $\operatorname{Diff}(M)$ of a compact manifold $M$ is known to be "large" in several senses. For example, it is relatively easy to show that if $\operatorname{dim}(M)>2$, then $\operatorname{Diff}(M)$ acts transitively on $k$-tuples of points in $M$ for every $k>0$. This makes the group $\operatorname{Diff}(M)$ seem relatively flexible, particularly in terms of the structure of its subgroups. Let $\operatorname{Diff}^{0}(M)$ be the connected component of $\operatorname{Diff}(M)$. A harder result to prove is that $\operatorname{Diff}^{0}(M)$ is topologically simple; i.e., any proper, closed normal subgroup is trivial [Ban]. This could be the beginning of a general structure theory of diffeomorphism groups, perhaps in analogy with Cartan's structure theory for simple Lie groups, but surprisingly little is known. Here we chose to explore $\operatorname{Diff}(M)$ by asking the following simple question about $\operatorname{Diff}(M)$.

Question 1.1. Given a compact manifold $M$, what finitely generated groups $\Gamma$ admit faithful homomorphisms $\rho$ : $\Gamma \rightarrow \operatorname{Diff}(M)$ ?

Question 1.1 has been asked before in many forms. The most classical topic here is the study of finite and, more

David Fisher is a professor of mathematics at Indiana University. His email address is fisherdm@indiana. edu.

Communicated by former Notices Associate Editor Bryna Kra.

For permission to reprint this article, please contact:

reprint-permission@ams.org.

DOI: https://doi.org/10.1090/noti2058 generally, compact groups of diffeomorphisms. This theory yields a rich set of topological obstructions to finding a finite group $F$ as a subgroup of $\operatorname{Diff}(M)$ or even of the homeomorphism group $\operatorname{Homeo}(M)$. In the area of research under discussion, we tend to focus on the case where $\Gamma$ is torsion free in order to generate new insights. In the broadest sense, the area is motivated by the idea that "large" groups of diffeomorphisms of compact manifolds should be of "geometric origin"; see particularly Gromov's article [Gro].

This article concerns a recent breakthrough due to Aaron Brown, the author, and Sebastian Hurtado on a conjecture of Zimmer's from 1983 [BFH1, BFH2, BFH3, Zim]. The conjecture concerns certain groups called higher rank lattices, which are described below in subsection 2.3. The conjecture predicts that these groups have no "interesting" actions on low-dimensional manifolds. For the original conjecture see [Zim, Conjecture II] and for a much more detailed unpacking of the conjecture see [Can, section 2.4]. To illustrate our work and Question 1.1, we state a special case of our results that proves a special case of the conjecture. This concerns the group $\operatorname{SL}(n, \mathbb{Z})$, the group of $n$ by $n$ integer matrices of determinant one.

Theorem 1.2 (Brown, Fisher, Hurtado). Let $M$ be a compact manifold and let $\rho: \operatorname{SL}(n, \mathbb{Z}) \rightarrow \operatorname{Diff}(M)$ be a homomorphism. Then:

1. if $\operatorname{dim}(M)<n-1$, the image of $\rho$ is finite;

2. if $\operatorname{dim}(M)<n$ and $\rho(\Gamma)$ preserves a volume form on $M$, then the image of $\rho$ is finite. 
Theorem 1.2 strongly restricts the actions of the "large" group $\operatorname{SL}(n, \mathbb{Z})$ on manifolds of small dimension. We will state a more general version below as Theorem 3.2, but do not go into details of the general conjecture or our most general results. The proofs of Theorems 1.2 and 3.2 bring together ideas from four areas:

1. rigidity theory;

2. smooth dynamics, particularly hyperbolic dynamics;

3. homogeneous dynamics, particularly the study of invariant measures;

4. operator algebras, particularly Lafforgue's strong prop$\operatorname{erty}(T)$.

The combination of ideas from diverse areas seems quite likely to lead to further dramatic progress on a wide variety of questions. Rigidity theory arises from the study of the geometry of locally symmetric spaces, and an iconic result is Mostow's rigidity theorem, which plays a key role in the study of topology of three-manifolds. Hyperbolic dynamics concerns dynamical systems where orbits diverge and converge quickly, exhibiting chaotic behavior. Homogeneous dynamics concerns special dynamical systems that are algebraically defined and frequently connect to number theory. Operator algebras arise out of the study of quantum mechanics, and the ideas used here arise from the study of $K$-theory and index theorems.

In this article, we will put Theorem 1.2 and the general conjecture in a broader context, state a more general case of our results formally, and then try to indicate how ideas from the four areas above contributed to the solution. First, we will describe a couple of key examples of actions of large groups on compact manifolds, both of geometric origin, and also give more examples of "large" groups. These examples help illustrate why Theorem 1.2 is natural.

\section{Examples and Motivation}

In this section, we provide examples and motivation. First we describe some natural group actions, then we describe some more general classes of "large" groups and state a more general form of Theorem 1.2. Finally, we give a very brief introduction to some classical results on rigidity of large groups that motivated Zimmer to conjecture Theorem 1.2.

2.1. First examples of group actions. In this section, we will describe two constructions of group actions. These serve two purposes. First, they show that the dimension bounds in Theorem 1.2 are sharp. Second, they illustrate that natural group actions often have geometric origin.

Projective actions. First consider the action of the projective linear group $\operatorname{PSL}(n, \mathbb{R})$ on the projective space $P\left(\mathbb{R}^{n}\right)$. For our purposes one can also consider the action of a double cover of $\operatorname{PSL}(n, \mathbb{R})$ on the $(n-1)$-sphere $S^{n-1}$. These actions are obtained by letting any invertible matrix act on lines or rays through the origin in $\mathbb{R}^{n}$. The scalar matrices act trivially on lines, so this defines an action of $\operatorname{PSL}(n, \mathbb{R})=\mathrm{GL}(n, \mathbb{R}) / D$ on $P\left(\mathbb{R}^{n}\right)$ where $D$ is the group of scalar matrices. If one wants the action on the sphere and so considers the action on rays, only the positive scalar matrices act trivially, and so one obtains an action of a slightly larger group. It is also clear that any subgroup of $\operatorname{PSL}(n, \mathbb{R})$ acts on $P\left(\mathbb{R}^{n}\right)$. It will be slightly more convenient later on to think of this as an action of $\operatorname{SL}(n, \mathbb{R})$ and its subgroups, in which case we can talk about the action on either $P\left(\mathbb{R}^{n}\right)$ or $S^{n-1}$. This therefore gives examples of actions of $\operatorname{SL}(n, \mathbb{Z})$ on manifolds of dimension $n-1$ and shows that the first conclusion of Theorem 1.2 is sharp. The action of $\operatorname{SL}(n, \mathbb{R})$ on either $P\left(\mathbb{R}^{n}\right)$ or $S^{n-1}$ preserves the natural projective geometry on the space; i.e., it sends (unparametrized) lines to (unparametrized) lines preserving incidence.

Affine actions. The second example is slightly more subtle. We have a natural action of $\operatorname{SL}(n, \mathbb{R})$ on $\mathbb{R}^{n}$, and if we restrict this to the integer matrices $\operatorname{SL}(n, \mathbb{Z})$, then the action preserves the integer lattice $\mathbb{Z}^{n}<\mathbb{R}^{n}$. This allows us to define an action of $\operatorname{SL}(n, \mathbb{Z})$ on the $n$ torus $\mathbb{T}^{n}=\mathbb{R}^{n} / \mathbb{Z}^{n}$. This action is different from the action on $P\left(\mathbb{R}^{n}\right)$ in two regards: first, it doesn't arise as the restriction of an action of $\operatorname{SL}(n, \mathbb{R})$, and second, it preserves the standard volume form on $\mathbb{T}^{n}$. As we will discuss at greater length later, $\operatorname{SL}(n, \mathbb{Z})$ is a lattice in $\operatorname{SL}(n, \mathbb{R})$. Both the group $\operatorname{SL}(n, \mathbb{Z})$ and this particular action have played an important role in the history of this field. This action shows that the second conclusion of Theorem 1.2 is sharp. This action preserves the natural affine geometry on the torus; i.e., it sends parametrized lines to parametrized lines preserving incidence.

Geometry of the actions. If we let $\operatorname{Aff}\left(\mathbb{T}^{n}\right)$ be the full group of affine diffeomorphisms of the torus and $\operatorname{Proj}\left(S^{n-1}\right)$ be the group of projective transformations of the sphere, we see another common element of these two actions. The action of $\operatorname{Aff}\left(\mathbb{T}^{n}\right)$ is transitive on $\mathbb{T}^{n}$, and the action of $\operatorname{Proj}\left(S^{n-1}\right)$ is transitive on $S^{n-1}$. This makes these manifolds into geometrically natural homogeneous spaces, and the actions of $\mathrm{SL}(n, \mathbb{Z})$ we define come as symmetries of these homogeneous geometric structures.

One can build many slightly more complicated variants of these actions by taking subgroups of $\mathrm{SL}(n, \mathbb{R})$ and letting them act either on the manifolds described above or on naturally defined submanifolds. In the next subsection we will describe some natural subgroups arising geometrically.

2.2. Large groups and geometry. We first consider subgroups of $\operatorname{SL}(n, \mathbb{R})$ that are defined as stabilizers of natural geometric objects on $\mathbb{R}^{n}$. For example, $\operatorname{SL}(n, \mathbb{R})$ is the subgroup of matrices preserving the standard volume form. 
Some other natural examples of subgroups include:

- the symplectic group $\operatorname{Sp}(2 n, \mathbb{R})$, which is the group preserving the natural symplectic form;

- the orthogonal group $O(p, q)$, which is the subgroup preserving the standard quadratic form of signature $(p, q)$ where $p+q=n$;

- the orthogonal group $O(n)$, which is the subgroup preserving a positive definite inner product on $\mathbb{R}^{n}$.

These groups are examples of a class known collectively as semisimple Lie groups. While most modern accounts begin very differently, the description just given is very much the classical one: semisimple lie groups are the linear groups that preserve some natural geometric structure on $\mathbb{R}^{n}$. This classical intuition holds for almost all semisimple Lie groups. There are finitely many exceptional groups where the connection to geometry is perhaps a bit less natural.

The second class of subgroups of $\operatorname{SL}(n, \mathbb{R})$ that will interest us is the discrete subgroups. We will actually be most interested in a special subclass of discrete subgroups, those that are lattices in the semisimple Lie groups described above. For now we will only say that the reader should keep the group $\operatorname{SL}(n, \mathbb{Z})$ in mind here.

Groups of symmetries play an important part in many areas of mathematics, with large groups of symmetries often indicating that objects have special properties. The simple and semisimple Lie groups are often paradigmatic examples of "large" symmetry groups. Closed connected subgroups of these groups are well understood, but their discrete subgroups remain more mysterious despite much study. Here we want to relate these to other, less studied "large" groups, like the group of diffeomorphisms $\operatorname{Diff}(M)$ of a compact manifold.

Just as we obtained subgroups of $\operatorname{SL}(n, \mathbb{R})$ by looking at subgroups preserving natural geometric objects, we can obtain subgroups of $\operatorname{Diff}(M)$ by looking at subgroups preserving a natural geometric structure. For example:

- the subgroup of diffeomorphisms preserving a volume form on $M$, the analogue of $\operatorname{SL}(n, \mathbb{R})<$ $\mathrm{GL}(n, \mathbb{R})$;

- the subgroup of diffeomorphisms preserving a symplectic form on $M$, the analogue of $\operatorname{Sp}(2 n, \mathbb{R})$;

- the subgroup of diffeomorphisms preserving a quadratic form of signature $(p, q)$ on $M$, i.e., the isometries of a pseudo-Riemannian metric on $M$, the analogue of $O(p, q)$;

- the subgroup of diffeomorphisms preserving a nondegenerate, positive, symmetric form, i.e., the isometries of a Riemannian metrc, the analogue of $O(n)$.

We will refer to diffeomorphisms preserving any geometric structure like those listed above as isometries of the geometric structure. The last example is very special, since the isometry group of a Riemannian metric on a compact manifold is always a compact Lie group. For some types of geometric structures, like a volume form or a symplectic form, the group of isometries remains infinite dimensional. For others, like a pseudo-Riemannian metric or an affine or projective connection, it is automatically finite dimensional. In this context, Gromov defined a notion of rigid geometric structure to try to capture the class of all geometric structures whose isometry groups are necessarily finite dimensional. A natural question raised independently and in slightly different forms by Gromov and Zimmer is whether any "large enough" subgroup of diffeomorphisms of a compact manifold necessarily preserves some natural rigid geometric structure on the manifold.

2.3. Lattices and classical rigidity results. An important, but somewhat indirect, impetus for the question of Gromov and Zimmer comes from the theory of discrete subgroups of Lie groups. A basic example of a discrete subgroup of a Lie group is the subgroup $\operatorname{SL}(n, \mathbb{Z})$ in $\operatorname{SL}(n, \mathbb{R})$. An interesting fact about this particular discrete subgroup is that the quotient space $\operatorname{SL}(n, \mathbb{R}) / \operatorname{SL}(n, \mathbb{Z})$ has finite volume. Given a Lie group $G$, a discrete subgroup $\Gamma<G$ is a lattice in $G$ if $G / \Gamma$ has finite Haar measure. An even more basic example, which already appeared above, is $\mathbb{Z}^{n}<\mathbb{R}^{n}$. The class of Lie groups most important for us here is simple Lie groups of real rank at least 2, and we refer to lattices in these groups as higher rank lattices. We do not define rank formally here, but point out that it is exactly the class of Lie groups whose lattices are known to have very strong rigidity properties, many of which we will encounter below. Paradigmatic examples are $\operatorname{SL}(n, \mathbb{R})$ when $n>2$ and $\operatorname{SL}(n, \mathbb{Z})$ again when $n>2$. A key motivation for the assumption that $n>2$ is that there are lattices in $\operatorname{SL}(2, \mathbb{R})$ that are free groups, and for the purposes of the question we study here, free groups do not appear to be "large." There are several senses in which a generic collection of $k$ diffeomorphisms of any compact manifold $M$ generates a free group $F_{k}$ inside $\operatorname{Diff}(M)$, and one cannot expect an invariant geometric structure of any kind this broadly. Free groups also do not satisfy any of the rigidity properties we will discuss below.

Two of the main rigidity properties of higher rank lattices are Margulis's superrigidity theorem, which classifies the linear representations of these groups, and Margulis's arithmeticity theorem, which uses the superrigidity theorem to classify the higher rank lattices. The arithmeticity theorem shows that all the lattices we consider here can be constructed by more complicated arithmetic analogues of taking the integer points of a group of matrices. The superrigidity theorem is harder to describe, so here we only mention a corollary that is a linear analogue of Theorems 1.2 and 3.2 below. 
Corollary 2.1 (Margulis). Let $\Gamma<\mathrm{SL}(n, \mathbb{R})$ be a lattice with $n>2$. Any homomorphism $\rho: \Gamma \rightarrow \mathrm{GL}(n-1, \mathbb{C})$ has finite image.

While Margulis's theorems sound algebraic in nature, Margulis's proof of his superrigidity theorem makes extensive use of dynamics and ergodic theory, providing a general impetus to study rigidity properties of these same groups in various dynamical settings. All known proofs of Margulis's superrigidity theorem use dynamical ideas in an essential way. This remains true even if one only wants to prove Corollary 2.1. If $\Gamma<\operatorname{SL}(n, \mathbb{R})$ is cocompact, then one can give a proof using harmonic map techniques instead, but there is no robust proof of an algebraic nature.

\section{The Zimmer Program}

In aiming to understand when large subgroups of diffeomorphism groups arose geometrically, Zimmer set the intermediate goal of trying to understand when higher rank lattices and even higher rank simple Lie groups can act on manifolds. Zimmer speculated that any action of these groups on a compact manifold arises geometrically. The collection of questions and results that arose from this goal are often referred to as the Zimmer program. A key impetus for Zimmer's speculation was his discovery of the cocycle superrigidity theorem, which showed that measurepreserving actions of these groups preserve certain kinds of "measurable geometric structures." We will not describe this result in general but will state an analogue of Corollary 2.1 that we will need later.

Corollary 3.1 (Zimmer). Let $\Gamma<\operatorname{SL}(n, \mathbb{R})$ be a lattice, let $n>2$, and let $M$ be a compact manifold of dimension at most $n-1$. Let $\rho: \Gamma \rightarrow \operatorname{Diff}(M)$ be a homomorphism. For any $\Gamma$ invariant measure $\mu$, the group $\rho(\Gamma)$ preserves a $\mu$-measurable Riemannian metric $\mathrm{g}$.

If one could prove that the Riemannian metric $g$ of Corollary 3.1 is smooth, then Corollary 3.1 together with a more precise form of Corollary 2.1 would imply Theorems 1.2 and 3.2. The point is that the isometry group of a smooth Riemannain metric is a compact Lie group and compact Lie groups are linear. The reader may then immediately wonder what a $\mu$-measurable Riemannian metric is. The simplest answer is that while a Riemannian metric is usually defined by a family $\left\{g_{i, j}\right\}$ of smooth functions that define a positive definite inner product at all points, here we relax that assumption to allow each $g_{i, j}$ to be a $\mu$ measurable function while still insisting that $\left\{g_{i, j}\right\}$ define a positive definite inner product at $\mu$ almost every point. Zimmer tended to emphasize the case where $\mu$ was volume and the $\Gamma$ action on $M$ was volume preserving, but the fact that Corollary 3.1 holds for all $\Gamma$ invariant measures is very important to recent developments. Even in the case when $\mu$ is a volume form, $g$ need not define a distance function on $M$. It may be the case that all curves between all pairs of points have infinite length! One might hope to find a way to see that $g$ is integrable along a good family of curves, but all known proofs of analogues of Corollary 3.1 produce metrics with either no or very weak integrability properties. This oddity has to do with the nature of the proof: it uses ideas from ergodic theory and makes almost no use of the smooth structure on $M$. We use Corollary 3.1 in our work, but rather than trying to improve regularity of $g$, we use an even weaker consequence described below in Corollary 4.2 .

In some special cases Zimmer could prove that $g$ was in fact smooth, and this led him to conjecture in 1983 that this was always the case. The conjecture was typically phrased in terms of a classification of low-dimensional actions, but the main impetus was the belief that $g$ was smooth. Brown, the author, and Hurtado have proven many cases of this conjecture and made substantial progress in all cases. The general conjecture and the full results with Brown and Hurtado require delving into the structure of simple Lie groups, but a more general version of Theorem 1.2 is:

Theorem 3.2 (Brown, Fisher, Hurtado). Let $\Gamma$ be a lattice in $\mathrm{SL}(n, \mathbb{R})$, let $M$ be a compact manifold, and let $\rho: \Gamma \rightarrow$ $\operatorname{Diff}(M)$ be a homomorphism. Then:

1. if $\operatorname{dim}(M)<n-1$, the image of $\rho$ is finite;

2. if $\operatorname{dim}(M)<n$ and $\rho(\Gamma)$ preserves a volume form on $M$, then the image of $\rho$ is finite.

This is more general than Theorem 1.2. Any finite index subgroup of $\operatorname{SL}(n, \mathbb{Z})$ is also a lattice, and $\operatorname{SL}(n, \mathbb{Z})$ has many finite index subgroups. One can build them as the kernels of a reduction $\bmod p$ map from $\operatorname{SL}(n, \mathbb{Z}) \rightarrow$ $\operatorname{SL}(n, \mathbb{Z} / p \mathbb{Z})$. There are also many other constructions of lattices in $\operatorname{SL}(n, \mathbb{R})$, producing a wide variety of groups with no finite index subgroups in common.

While Theorem 3.2 says that the resulting group action has to factor through a finite quotient of $\Gamma$, one should notice that this implies there is an invariant smooth Riemannian metric. Simply take any smooth Riemannian metric and average its translates under $\rho(\Gamma)$. Really, the proof passes through the existence of an invariant smooth Riemannian metric for $\rho(\Gamma)$ and so is very much along the lines of showing that "large" symmetry groups of compact manifolds preserve geometric structures.

\section{Ideas in the Proof of Theorem 3.2}

The major pivot point of the proof is establishing that derivatives of the $\Gamma$ action grow subexponentially. To begin describing this, we start in the simpler setting of linear algebra and progress to dynamical settings with the linear model in mind. 
Given an $n \times n$ matrix $A$ and a vector $v$, it is easy to see that the norm of $A^{n} v$ grows more slowly than $\lambda^{n}$ where $\lambda=\|A\|$ is the largest absolute value of an eigenvalue of $A$, provided $\lambda>1$. If all eigenvalues are of unit modulus, then the norm of $A^{n} v$ grows polynomially in $n$. The exponential growth is typically called hyperbolic behavior, while the polynomial growth is called parabolic behavior. The analogues of these notions in the setting of diffeomorphisms of manifolds are much more complicated. If $f: M \rightarrow M$ is a diffeomorphism and $v_{x}$ is a vector at a point $x$, then $D\left(f^{n}\right)_{x} v_{x}$ is, by the chain rule, a composition of matrices defined by taking derivatives of $f$ along the $f$ orbit of $x$. In some special cases, this can be modelled well as a product of random matrices. Random models typically show nice behavior generically. However, the information provided by random models is often insufficient to solve dynamical problems.

In the context of Theorem 3.2, it follows from Corollary 3.1 that the action is almost parabolic generically. By this we mean that the norms of derivatives grow subexponentially at random points. The bulk of the work in our proof of Theorem 3.2 is improving the sense in which derivatives grow subexponentially, from a random or generic sense to a uniform sense. Once we can show that derivatives grow subexponentially, the rest of our proof is mainly an application of Lafforgue's strong property $(T)$ combined with some ideas on averaging Riemannian metrics originating in the author's work with Margulis [FM].

4.1. From random estimates to deterministic estimates. To make the above discussion precise requires some definitions. The following is an ad hoc variant of a standard definition in the theory of dynamical systems. We first note that lattices in $\operatorname{SL}(n, \mathbb{R})$ are finitely generated and so admit a word length, which we write as $l: \Gamma \rightarrow \mathbb{N}$. The probabilistic or generic subexponential growth referred to above is captured in the following definition.

Definition 4.1. Let $\rho: \Gamma \rightarrow \operatorname{Diff}(M)$ be an action of a finitely generated group on a compact manifold. Let $\mu$ be a measure on $M$. We say $\rho$ has zero first Lyapunov exponent for $\mu$ if

$$
\lim _{l(\gamma) \rightarrow \infty} \frac{\ln \|D \rho(\gamma)(x)\|}{l(\gamma)}=0
$$

for $\mu$ almost every $x$ in $M$.

The reader may be slightly unsure why we refer to this as a probabilistic definition but should note that the measure $\mu$ plays a key role. The estimate holds only for almost every point of $\mu$, and the rate at which the limit converges is also essentially random both in $x$ and $\Gamma$. This is a very weak form of subexponential growth, happening only almost everywhere for some measure and at a rate that can depend on both the point in $M$ and the sequence of elements going to infinity in $\Gamma$. We now state a consequence of Corollary
3.1 that shows this slow rate of growth holds for any $\Gamma$ invariant measure $\mu$ in the context of Theorem 3.2.

Corollary 4.2 (Zimmer). Let $\Gamma<\operatorname{SL}(n, \mathbb{R})$ be a lattice, let $n>2$, and let $M$ be a compact manifold of dimension at most $n-1$. Let $\rho: \Gamma \rightarrow \operatorname{Diff}(M)$ be a homomorphism. Then $\rho$ has zero first Lyapunov exponent for any $\rho(\Gamma)$ invariant measure $\mu$.

A major difficulty occurs at this point in the proof in that it is a priori completely unclear that there are any invariant measures for the $\Gamma$ action on $M$. Nevo and Zimmer had previously proved that either an invariant measure exists or the action had a measurable projective factor [NZ]. This condition for the existence of an invariant measure was essentially uncheckable in practice. A different approach taken by Brown, Rodriguez Hertz, and Wang produced an invariant measure for the $\Gamma$ action exactly in the low dimensions relevant to Theorem 3.2 or Corollary 3.1, and techniques from their proof provide a key ingredient in ours [BRHW]. To describe the next key step in our work requires another definition. Recall that $l$ is a length function on $\Gamma$ given by word length.

Definition 4.3. Let $\rho: \Gamma \rightarrow \operatorname{Diff}(M)$ be an action of a finitely generated group $\Gamma$ on a compact manifold $M$. We say $\rho$ has subexponential growth of derivatives if for every $\epsilon>0$ there exists $C>0$ such that

$$
\sup _{x \in M}\left\|D \rho(\gamma)_{x}\right\|<C e^{\varepsilon l(\gamma)} .
$$

This definition says that the norm of the derivative of $\rho$ grows slower than any exponential function. It is most important for us here that the estimate is uniform over the entire manifold and does not depend in any way on the basepoint or an invariant measure. The reader should note that this statement is still much weaker than saying derivatives grow at a polynomial rate, but it turns out to be sufficient for our purposes due to deep results of Lafforgue, which are discussed below.

The most difficult step in the proof of Theorem 3.2 is the following result.

Theorem 4.4 (Brown-Fisher-Hurtado). Let $\Gamma$ be a lattice in $\operatorname{SL}(n, \mathbb{R})$, let $M$ be a compact manifold, and let $\rho: \Gamma \rightarrow$ $\operatorname{Diff}(M)$ be a homomorphism.

1. If $\operatorname{dim}(M)<n-1$, then $\rho$ has subexponential growth of derivatives.

2. If $\operatorname{dim}(M)<n$ and $\rho(\Gamma)$ preserves a volume form on $M$, then $\rho$ has subexponential growth of derivatives.

We motivate the connection between Theorem 4.4 and Corollary 4.2 by the following classical proposition.

Proposition 4.5. Let $M$ be a compact manifold and let $\rho$ : $\mathbb{Z} \rightarrow \operatorname{Diff}(M)$ be the action generated by a single diffeomorphism $f$. Then $\rho$ has subexponential growth of derivatives if and only if $\rho$ has zero first Lyapunov exponent for every $f$ invariant measure $\mu$. 
The proof of this proposition is quite easy and involves a simple construction of invariant measures. If one has a diffeomorphism $f$ one can look at averages of Dirac measures over $f$ orbits, i.e.,

$$
\mu_{n, x}=\frac{1}{n+1} \sum_{i=0}^{n} \delta_{f^{i}(x)} .
$$

Measures of this kind are often referred to in the literature as empirical measures. Any collection $\left\{\mu_{n, x_{n}}\right\}$ has weak-* limits by compactness of $M$, and it is easy to check that the limiting measure is $f$ invariant. The point is that $f_{*} \mu_{n, x}$ differs from $\mu_{n, x}$ only by its values at "the boundary" of the interval $[0, n+1]$, i.e., only at the points 0 and $n+1$, and each of these points has mass going to zero with $n$. To show that any action that fails to have subexponential growth of derivatives has an invariant measure that doesn't have zero first Lyapunov exponent, one constructs empirical measures based at points that witness the exponential growth of derivatives. Any weak-* limit of a sequence of such measures can be shown to have positive first Lyapunov exponent.

The difficulty in mimicking this proof for a group like $\mathrm{SL}(n, \mathbb{Z})$ or $\mathrm{SL}(n, \mathbb{R})$ comes at the step of producing limits of empirical measures. One might think that one can define analogues of the $\mu_{n, x}$ by, say, averaging Dirac measures against larger and larger balls in any finitely generated group $\Gamma$. While one can define such measures and take their weak-* limits, it turns out that these are $\Gamma$ invariant if and only if $\Gamma$ is amenable, i.e., only if one can find a sequence of larger and larger sets in $\Gamma$ whose boundary is "relatively small." In fact, von Neumann originally defined amenable groups to be groups for which any action on a compact space had an invariant measure. It is well known that higher rank simple groups and their lattices are not amenable.

Overcoming this difficulty is possible but requires a large detour in the proof of Theorem 4.4, and the bulk of the work in [BFH1] occurs here. In particular the proof of Theorem 4.4 mixes techniques and ideas from nonuniformly hyperbolic dynamics, homogeneous dynamics, and classical rigidity theory. This combination of ideas seems promising for pursuing myriad other questions related to group actions on manifolds. I will give a brief overview of the proof of Theorem 4.4, only to point to various ingredients and where they come from.

4.2. Induced actions and proof outline for Theorem 4.4. The first step in the proof of Theorem 4.4 uses the notion of an induced action, a variant of induced representations due to Mackey. This notion is also similar to the construction of flat bundles. If $\Gamma$ acts on a manifold $M$ via a homomorphism $\rho: \Gamma \rightarrow \operatorname{Diff}(M)$, then we can build a $G$ action on a manifold $(G \times M) / \Gamma$. This can be specified just by specifying commuting $G$ and $\Gamma$ actions on $G \times M$. We do this by the formula

$$
g\left(g_{0}, m\right) \gamma=\left(g g_{0} \gamma^{-1}, \rho(\gamma) m\right)
$$

Note that there is a $G$ equivariant map $\pi:(G \times M) / \Gamma \rightarrow$ $G / \Gamma$, and this map exhibits $(G \times M) / \Gamma$ as a fiber bundle over $G / \Gamma$ with fiber $M$. Also note that the tangent bundle to $(G \times M) / \Gamma$ admits a $G$ invariant subbundle consisting of directions tangent to fibers of the projection $\pi$, i.e., $(G \times$ $T M) / \Gamma \subset T(G \times M) / \Gamma$.

We then define fiberwise zero first Lyapunov exponent and fiberwise subexponential growth of derivatives for the $G$ action on $(G \times M) / \Gamma$ by restricting all derivatives in the definitions to the invariant subbundle $(G \times T M) / \Gamma \subset T(G \times M) / \Gamma$. It is a relatively easy exercise to see that if $\Gamma<G$ is cocompact, then subexponential growth of derivatives for the $\Gamma$ action is equivalent to fiberwise subexponential growth of derivatives for the $G$ action on $(G \times M) / \Gamma$. The situation when $G / \Gamma$ has finite volume but is not compact is considerably more complicated, and we do not discuss it here. At this point, the structure of Lie groups begins to play an important role. It turns out that $G$ can always be written as a product $K A K$ where $K$ is compact and $A$ is abelian. For $\mathrm{SL}(n, \mathbb{R})$, these groups are $K=\mathrm{SO}(n)$ and $A$ is the group of diagonal matrices of determinant one. Since $K$ is compact, we can average any Riemannian metric on $(G \times M) / \Gamma$ over the $K$ action and obtain a $K$ invariant metric. This means that for the action of $G$ any growth of derivatives that we see comes entirely from the action of $A$. Modifying the proof of Proposition 4.5 and retaining the notation and terminology above, we prove the following.

Lemma 4.6. Given $\rho: \Gamma \rightarrow \operatorname{Diff}(M)$ either $\rho$ has subexponential growth of derivatives or there is an $A$ invariant measure $\mu$ on $(G \times M) / \Gamma$ without fiberwise zero first Lyapunov exponent for some element a in $A$.

If $\mu$ were in fact $G$ invariant, then this could be seen to contradict Corollary 4.2. The key point that makes it possible to prove Lemma 4.6 is that $A$ is abelian and so amenable. This amenability is just the familiar fact that the surface area of a sphere grows more slowly than the volume of the ball it encloses.

We proceed by proving that $\mu$ can be replaced by a measure that is in fact $G$ invariant. This is done in two steps. First we average the measure over certain subgroups of $G$ to produce a measure $\mu^{\prime}$ whose projection $\pi_{*} \mu^{\prime}$ to $G / \Gamma$ is Haar measure. The difficulty here is to do the averaging while retaining that $\mu^{\prime}$ is $A$ invariant and that some $a$ in $A$ has positive first Lyapunov exponent for $\mu^{\prime}$. After this step, we can use a result of Brown, Rodriguez Hertz, and Wang together with some algebraic computations to show that $\mu^{\prime}$ is in fact $G$ invariant [BRHW]. This contradiction shows that $\rho$ does in fact have subexponential growth of derivatives. 
The step of averaging the measure $\mu$ to produce $\mu^{\prime}$ makes extensive use of homogeneous dynamics and, in particular, work of Ratner and Shah [Rat1, Rat2, Sha]. The work of Brown, Rodriguez Hertz, and Wang pivots on relations between invariant measures and entropy and in particular on an extension of the important work of Ledrappier and Young [LY]. A key ingredient in both parts is the theory of Lyapunov exponents and particularly the fact that for actions of an abelian group $A$, Lyapunov exponents give rise to linear functionals on $A$.

4.3. Strong property $(T)$ and invariant metrics. Once we have established Theorem 4.4, the remaining step in the proof of Theorem 3.2 is to use Lafforgue's strong property $(T)$ to find a $\Gamma$ invariant metric. This is quite surprising, as Lafforgue introduced this property to study deep conjectures concerning $K$-theory of operator algebras. The other applications of the property to date have concerned geometry of Banach spaces and theory of computation.

It is known by results of Lafforgue, de Laat, and de la Salle that strong property $(T)$ holds for all higher rank lattices in the sense of this article [Laf, dLdlS, dlS]. Therefore Theorem 4.4 combined with Theorem 4.7 below immediately gives an invariant smooth Riemannian metric in the context of Theorem 3.2. The definition of strong property $(T)$ is already quite involved and uses either several quantifiers or the introduction of certain operator algebraic notions, so we do not give it here. Instead we mention that it is a strengthening of Kazhdan's property $(T)$. The simplest definition of property $(T)$ is that a group $\Gamma$ has property $(T)$ if every isometric action of $\Gamma$ on a Hilbert space fixes a point. This means that all isometric actions are conjugate to unitary representations.

We summarize this step in the proof of Theorem 3.2 in the following theorem.

Theorem 4.7 (Brown, Fisher, Hurtado). Let $\Gamma$ be a group with the strong property $(T)$ of Lafforgue. Let $M$ be a compact manifold and let $\rho: \Gamma \rightarrow \operatorname{Diff}(M)$ be an action of $\Gamma$ that has subexponential growth of derivatives. Then $\rho(\Gamma)$ preserves a smooth Riemannian metric on $M$.

We indicate the ideas in the proof of Theorem 4.7 and the importance of strong property $(T)$ by contrasting the theorem with an earlier result of the author and Margulis [FM].

Theorem 4.8 (Fisher, Margulis). Let $\Gamma$ be a group with property $(T)$ of Kazhdan, let $M$ be a compact manifold, and let $\rho: \Gamma \rightarrow \operatorname{Isom}(M)$ be an isometric action. Then any action $\rho^{\prime}: \Gamma \rightarrow \operatorname{Diff}(M)$ close enough to $\rho$ also preserves a smooth Riemannian metric.

In fact, the author and Margulis prove the stronger result that $\rho^{\prime}$ is conjugate to $\rho$, but the proof of this weaker result is closer to the proof of Theorem 4.7. We now sketch the common outline of the proofs of Theorems 4.7 and 4.8. Choose a finitely supported measure $\mu$ on $\Gamma$, say $\mu=\frac{1}{|S|} \sum_{s \in S} \delta_{S}$, for some finite generating set $S$ of $\Gamma$. Choose a smooth metric $g_{0}$ on $M$ and consider $g_{n}=\int_{\Gamma} \rho(s)^{*} g_{n-1} d \mu(s)$, the sequence of metrics given by repeated averaging of $g_{0}$ with respect to $\mu$ and $\rho$. One then uses either property $(T)$ or strong property $(T)$ together with some dynamical estimates and some Sobolev theory to prove that $g_{n}$ converge to a smooth metric $g_{\infty}$ that is $\Gamma$ invariant.

A key difference between the proofs of Theorems 4.7 and 4.8 is in the control on derivatives given by the hypothesis. The hypotheses of Theorem 4.8 imply that there is an $\epsilon$ (depending on how close $\rho^{\prime}$ is to $\rho$ ) such that

$$
\sup _{x \in M}\|D \rho(\gamma)\| \leq e^{\epsilon l(\gamma)} .
$$

In other words, the assumption implies immediate slow exponential growth of derivatives. The presence of the constant $C$ in Definition 4.3 means that our definition of subexponential growth of derivatives is really about eventual slow exponential growth of derivatives, meaning in particular that the derivatives can have very large norm for small elements of $\Gamma$.

\section{Concluding Remarks}

While Theorem 3.2 is a major breakthrough in the Zimmer program, many interesting questions remain open. While Theorem 3.2 is sharp for lattices in $\operatorname{SL}(n, \mathbb{R})$ and the proof also provides sharp results for $\operatorname{Sp}(2 n, \mathbb{R})$, for all other simple groups $G$, the results we prove are not sharp. In some sense our results are worse for the finite collection of exceptional simple groups and worst for the largest one, $E_{8}$. But the larger question that remains broadly open, even for lattices $\Gamma<\operatorname{SL}(n, \mathbb{R})$, is classifying actions in dimensions greater than those understood by Theorem 3.2. A number of striking results have been proven where the dimension is arbitrary but always with some additional geometric or dynamical restriction on the action. The proof of Theorem 3.2 gives hope that unrestricted results might begin to be accessible in higher dimensions as well.

To close, we describe a key obstruction to further progress in the Zimmer program. There are examples of $n$-dimensional manifolds whose symmetry group is a lattice in $\operatorname{SL}(n, \mathbb{R})$ but which do not admit a rigid geometric structure. The simplest example is obtained by taking the $\operatorname{SL}(n, \mathbb{Z})$ action on $\mathbb{T}^{n}$ and blowing up the origin in the standard algebro-geometric sense. This example was first pointed out by Katok and Lewis, and the fact that it does not admit a rigid geometric structure was proven by Benveniste and the author $[\mathrm{KL}, \mathrm{BF}]$. Benveniste and the author do introduce a different type of geometric structure that is preserved. The techniques used to prove Zimmer's 
conjecture point to various approaches to classifying actions of $\operatorname{SL}(n, \mathbb{Z})$ on manifolds in dimension $n$, but all require something new to deal with the issues raised by exactly these examples. Very recently, the author has discovered some new and distinct constructions of actions of both $\operatorname{SL}(n, \mathbb{R})$ and $\operatorname{SL}(n, \mathbb{Z})$ on manifolds in dimension $n$. All of these exhibit structure similar to the Katok-Lewis examples in that they are formed by taking connected sums over invariant sets and the interior of the "pieces" are all homogeneous spaces with natural geometric structures. These geometric structures appear to degenerate in a manner similar to the examples of Katok and Lewis.

Remark. We add some suggestions for further reading here, where the interested reader can find more detailed bibliographies. For a fairly gentle and detailed overview of the Zimmer program from 2011, see [Fis1]. In particular, [Fis1] points to numerous other problems and directions of research not touched on here. For a more technical update focusing on the developments leading up to the results described here, see [Fis2]. For a more detailed introduction to some aspects of our proofs, see [Bro] or [Can]. For a full account of proofs, see the original articles [BFH1, BFH2, BFH3].

\section{References}

[Ban] Augustin Banyaga, The structure of classical diffeomorphism groups, Mathematics and its Applications, vol. 400, Kluwer Academic Publishers Group, Dordrecht, 1997. MR1445290

$[\mathrm{BF}]$ E. Jerome Benveniste and David Fisher, Nonexistence of invariant rigid structures and invariant almost rigid structures, Comm. Anal. Geom. 13 (2005), no. 1, 89-111. MR2154667

[Bro] A. Brown, Entropy, Lyapunov exponents, and rigidity of group actions, arXiv:1809.09192 2018. Ensaios Math. (to appear); Appendices by several authors.

[BFH1] A. Brown, D. Fisher, and S. Hurtado, Zimmer's conjecture: Subexponential growth, measure rigidity, and strong property (T), 2016.

[BFH2] A. Brown, D. Fisher, and S. Hurtado, Zimmer's conjecture for actions of $\operatorname{SL}(m, \mathbb{Z}), 2017$.

[BFH3] A. Brown, D. Fisher, and S. Hurtado, Zimmer's conjecture for non-uniform lattices and escape of mass, 2019.

[BRHW] A. W. Brown, F. Rodriguez Hertz, and Z. Wang, Invariant measures and measurable projective factors for actions of higher-rank lattices on manifolds, 2016.

[Can] S. Cantat, Progrès récents concernant le programme de Zimmer, Séminaire Bourbaki, Exposé 1136, Octobre 2017.

[dlS] Mikael de la Salle, Strong property $(T)$ for higher-rank lattices, Acta Math. 223 (2019), no. 1, 151-193, DOI 10.4310/ACTA.2019.v223.n1.a3, MR4018265

[dLdlS] Tim de Laat and Mikael de la Salle, Strong property ( $T$ ) for higher-rank simple Lie groups, Proc. Lond. Math. Soc. (3) 111 (2015), no. 4, 936-966, DOI 10.1112/plms/pdv040 MR3407190

[Fis1] David Fisher, Groups acting on manifolds: around the Zimmer program, Geometry, rigidity, and group actions, Chicago Lectures in Math., Univ. Chicago Press, Chicago, IL, 2011, pp. 72-157, DOI 10.7208/chicago/9780226237909.001.0001. MR2807830

[Fis2] D. Fisher, Recent progress on Zimmer's conjecture, 2017.

[FM] David Fisher and Gregory Margulis, Almost isometric actions, property $(T)$, and local rigidity, Invent. Math. 162 (2005), no. 1, 19-80, DOI 10.1007/s00222-004-0437-5 MR2198325

[Gro] Michael Gromov, Rigid transformations groups, Géométrie différentielle (Paris, 1986), Travaux en Cours, vol. 33, Hermann, Paris, 1988, pp. 65-139. MR955852

[KL] A. Katok and J. Lewis, Global rigidity results for lattice actions on tori and new examples of volumepreserving actions, Israel J. Math. 93 (1996), 253-280, DOI 10.1007/BF02761106 MR1380646

[Laf] Vincent Lafforgue, Un renforcement de la propriété $(T)$ (French, with English and French summaries), Duke Math. J. 143 (2008), no. 3, 559-602, DOI 10.1215/001270942008-029 MR2423763

[LY] F. Ledrappier and L.-S. Young, The metric entropy of diffeomorphisms. I. Characterization of measures satisfying Pesin's entropy formula, Ann. of Math. (2) 122 (1985), no. 3, 509539, DOI $10.2307 / 1971328$ MR819556

[NZ] Amos Nevo and Robert J. Zimmer, A structure theorem for actions of semisimple Lie groups, Ann. of Math. (2) 156 (2002), no. 2, 565-594, DOI 10.2307/3597198 MR1933077

[Rat1] Marina Ratner, On Raghunathan's measure conjecture, Ann. of Math. (2) 134 (1991), no. 3, 545-607, DOI 10.2307/2944357 MR1135878

[Rat2] Marina Ratner, Raghunathan's topological conjecture and distributions of unipotent flows, Duke Math. J. 63 (1991), no. $1,235-280$, DOI 10.1215/S0012-7094-91-06311-8 MR1106945

[Sha] Nimish A. Shah, Limit distributions of polynomial trajectories on homogeneous spaces, Duke Math. J. 75 (1994), no. 3, 711-732, DOI 10.1215/S0012-7094-94-07521-2 MR1291701

[Zim] Robert J. Zimmer, Actions of semisimple groups and discrete subgroups, Proceedings of the International Congress of Mathematicians, Vol. 1, 2 (Berkeley, Calif., 1986), Amer. Math. Soc., Providence, RI, 1987, pp. 1247-1258. MR934329

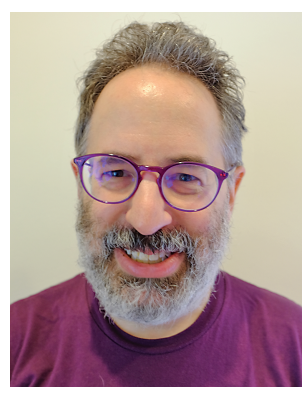

David Fisher

\section{Credits}

Opener image is courtesy of Getty. Photo of the author is by Charles Brandt. 